Introduction

Le décloisonnement des disciplines est une façon de favoriser la capacité, pour l'élève, de faire des liens entre les savoirs et de transférer ses apprentissages. La même logique prévaut au sein d'une discipline orchestrée autour du développement de compétences multiples, ces dernières étant souvent abordées distinctement.

En français, par exemple, la grammaire est traditionnellement enseignée à part des compétences à lire ou à écrire. Qui plus est, les interventions sont souvent les mêmes : l'enseignant donne une leçon - qu'il souhaite la plus dynamique et active possible! - et la complémente d'exercices cernant spécifiquement l'aspect langagier enseigné. Si de telles pratiques sont essentielles, surtout en début de parcours scolaire, on peut se questionner sur leur efficacité pour le développement de la compétence scripturale.

Une autre possibilité consiste à articuler langue et écriture en classe. L'articulation se réalisant tant par la planification, le pilotage et l'évaluation d'activités ou de séquences, elle est tributaire des choix de l'enseignant.

Après avoir mené une recension d'écrits sur l'articulation entre la grammaire et les compétences discursives ${ }^{1}$, nous en arrivons à quelques constats et recommandations issues des larges consensus. Comme montré à la figure 1 , ceux-ci s'organisent autour de deux plans : la macroplanification et la microplanification. 


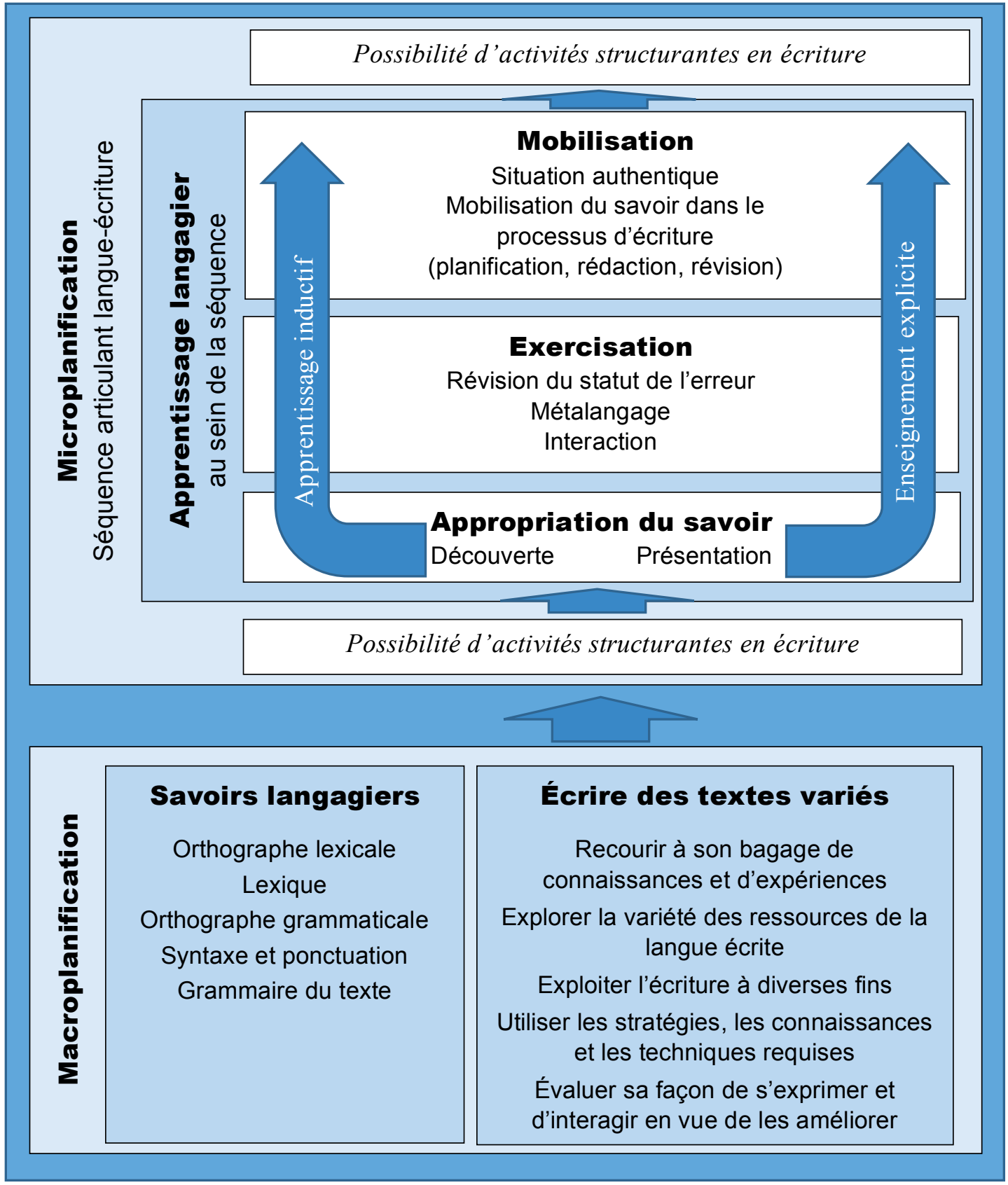

Figure 1

Planifier en articulant langue et écriture. 


\section{La macroplanification}

Pour s'assurer d'une articulation, il est nécessaire que la progression des savoirs des deux domaines soit prévue sur l'année selon leur interrelation. Au niveau secondaire, cela est facilité par une prescription qui l'explicite, en associant des savoirs grammaticaux à des genres textuels ou à des modes de discours qui l'exploitent particulièrement. Par exemple, les procédés de reprise tels que la répétition de mots ou de structures sont associés à l'enseignement du texte poétique, qui fait usage de ces procédés.

\section{La microplanification}

La microplanification est idéalement organisée en séquences ${ }^{2}$ Ici, les différentes activités - à la fois discursives et langagières - se nourrissent l'une et l'autre et mènent à une production authentique, c'est-à-dire à une tâche ayant une finalité de communication réelle. Elle permet alors à l'élève de mobiliser le savoir nouvellement acquis en savoir-faire et donne du sens aux objets d'enseignement.

Plutôt que d'aborder la grammaire distinctement, la planification en séquences permet de véritablement assurer des allers-retours entre les composantes - du français ou d'une autre discipline, chacune orchestrée autour d'un but commun. C'est ainsi qu'une séquence peut impliquer des tâches de lecture et d'écriture - et pourquoi pas à propos d'un sujet abordé en histoire ou en science! - et intégrer certaines activités grammaticales structurantes. Les activités dites structurantes visent à isoler l'apprentissage d'un objet de savoir grammatical qui, en contexte d'écriture, impliquerait une surcharge cognitive.

Deux modèles d'intervention sont principalement mis de l'avant, soit l'apprentissage inductif, qui favorise l'apprentissage par la découverte, ou encore explicite, qui vise une présentation par l'enseignant, laquelle sera suivie d'une mobilisation de plus en plus autonome par l'élève. Dans un cas comme dans l'autre, après une première phase d'appropriation et une exercisation, on doit réinvestir activement et progressivement dans une situation d'écriture prévue dans la séquence.

Pour l'exercisation, certaines activités offrent des résultats significativement supérieurs, comme les dictées innovantes (dictée zéro faute, dictée négociée, atelier de négociation graphique). De telles activités permettent aussi de dynamiser l'enseignement de la grammaire en l'appréhendant sous l'angle d'une résolution de problème.

Pour la mobilisation des savoirs grammaticaux lors des situations d'écriture, on remarque un certain consensus scientifique : pour dépasser un plateau, ils doivent être mobilisés de manière explicite. Cette mise en évidence se trouve facilitée par l'articulation au sein même de la planification puisqu'elle garantit une proximité entre le savoir grammatical et la compétence scripturale. Le travail collaboratif à différentes étapes du processus d'écriture (par exemple à la planification, par la production en équipe d'un champ lexical, ou à la révision, par une autocorrection de savoirs ciblés) est une des pistes ayant montré son efficacité, car elle permet la mobilisation du savoir grammatical dans une situation problème authentique, et nécessite l'utilisation du métalangage par les élèves. Le statut même de l'erreur est alors remis en question : elle devient le point de départ d'une réflexion langagière faisant appel aux savoirs préalablement acquis. Il s'agit encore une fois de permettre à l'élève de créer des liens entre les savoirs langagiers, et à réfléchir à leur place dans sa propre pratique de scripteur. 


\section{Conclusion}

Il est souvent mentionné, surtout au niveau secondaire, les limites organisationnelles relatives au décloisonnement des disciplines. Mais au sein d'une discipline unique, on peut se demander pourquoi langue et écriture sont si souvent abordées distinctement: simplement par tradition pédagogique ou par peur de «contaminer» les activités d'écriture par d'autres, souvent vues avec raison comme démotivantes? Inversons la réflexion et faisons le pari que d'une part, l'articulation bonifie la perception de valeur des savoirs langagiers, et que d'autre part, en écriture, la perception de contrôlabilité des élèves augmente, offrant aux élèves des ressources langagières concrètes pour rédiger et pour réfléchir, ce qui est essentiel dans toutes les disciplines.

\section{Notes}

1 Vincent, F., Émery-Bruneau, J., Dezutter, O., Lefrançois, P. et Larose, F. (2016). Lénseignement de la grammaire au service du développement de compétences en lecture et en écriture : une synthèse des connaissances, FRQSC 2014-2016.

2 Pour des exemples d'articulation lecture-écriture-grammaire (ALEG), voir le site w4.uqo.ca/aleg

\section{Pour citer cet article}

Vincent, F. et Leclerc, C. S. (2018). Articuler langue et écriture : un travail de planification... signifiant! Formation et profession, 26(1), 125-128. http://dx.doi.org/10.18162/fp.2018.a139 